\title{
ON THE GLOBAL CHARACTER OF THE RATIONAL
}

$$
\begin{gathered}
x_{n+1}=\frac{\alpha_{1}}{A_{1}+B_{1} x_{n}+y_{n}} \quad \text { AYSTEM } \\
\text { E. DRYMONIS AND G. LADAS }
\end{gathered}
$$

This paper is dedicated to Professor Mustafa Kulenovic on the occasion of his 60th birthday

\begin{abstract}
In this paper we investigate the global stability character of the rational system in the title with the parameters $B_{1}, B_{2}, A_{2}+C_{2}$ positive, the parameters $A_{1}, \alpha_{2}, \beta_{2}, A_{2}, C_{2}$ nonnegative, and with arbitrary nonnegative initial conditions such that the denominators are always positive.
\end{abstract}

\section{Introduction and Preliminaries}

In this paper we investigate the global stability character of the rational system

$$
x_{n+1}=\frac{\alpha_{1}}{A_{1}+B_{1} x_{n}+y_{n}} \text { and } y_{n+1}=\frac{\alpha_{2}+\beta_{2} x_{n}}{A_{2}+B_{2} x_{n}+C_{2} y_{n}}, \quad n=0,1, \ldots,
$$

with

$$
B_{1}, B_{2}, A_{2}+C_{2}>0 \text { and } A_{1}, \alpha_{2}, \beta_{2}, A_{2}, C_{2} \geq 0
$$

and with arbitrary nonnegative initial conditions $x_{0}$ and $y_{0}$ such that the denominators are always positive.

In the notation that was introduced in [12], System (1.1) contains the following 18 special cases:

$$
\begin{aligned}
& \#(12,11): \quad x_{n+1}=\frac{\alpha_{1}}{x_{n}+y_{n}}, \quad y_{n+1}=\frac{\alpha_{2}}{1+x_{n}} \\
& \#(37,11): \quad x_{n+1}=\frac{\alpha_{1}}{A_{1}+x_{n}+y_{n}}, \quad y_{n+1}=\frac{\alpha_{2}}{1+x_{n}}
\end{aligned}
$$

2000 Mathematics Subject Classification. 39A10.

Key words and phrases. Boundedness, boundedness characterization, global stability, periodicity, patterns of boundedness, rational difference equations, rational systems. 


$$
\begin{aligned}
& \#(12,12): x_{n+1}=\frac{1}{B_{1} x_{n}+y_{n}}, \quad y_{n+1}=\frac{1}{B_{2} x_{n}+y_{n}} \\
& \#(37,12): x_{n+1}=\frac{1}{A_{1}+B_{1} x_{n}+y_{n}}, \quad y_{n+1}=\frac{1}{B_{2} x_{n}+y_{n}} \\
& \#(12,17): x_{n+1}=\frac{\alpha_{1}}{x_{n}+y_{n}}, \quad y_{n+1}=\frac{x_{n}}{A_{2}+x_{n}} \\
& \#(37,17): x_{n+1}=\frac{\alpha_{1}}{A_{1}+x_{n}+y_{n}}, \quad y_{n+1}=\frac{x_{n}}{A_{2}+x_{n}} \\
& \#(12,18): x_{n+1}=\frac{\alpha_{1}}{x_{n}+y_{n}}, \quad y_{n+1}=\frac{x_{n}}{B_{2} x_{n}+y_{n}} \\
& \#(37,18): x_{n+1}=\frac{\alpha_{1}}{A_{1}+x_{n}+y_{n}}, \quad y_{n+1}=\frac{x_{n}}{B_{2} x_{n}+y_{n}} \\
& \#(12,32): x_{n+1}=\frac{\alpha_{1}}{x_{n}+y_{n}}, \quad y_{n+1}=\frac{\alpha_{2}+\beta_{2} x_{n}}{1+x_{n}} \\
& \#(37,32): x_{n+1}=\frac{\alpha_{1}}{A_{1}+x_{n}+y_{n}}, \quad y_{n+1}=\frac{\alpha_{2}+\beta_{2} x_{n}}{1+x_{n}} \\
& \#(12,33): x_{n+1}=\frac{1}{x_{n}+y_{n}}, \quad y_{n+1}=\frac{\alpha_{2}+\beta_{2} x_{n}}{B_{2} x_{n}+y_{n}} \\
& \#(37,33): x_{n+1}=\frac{1}{A_{1}+x_{n}+y_{n}}, \quad y_{n+1}=\frac{\alpha_{2}+\beta_{2} x_{n}}{B_{2} x_{n}+y_{n}} \\
& \#(12,37): x_{n+1}=\frac{\alpha_{1}}{x_{n}+y_{n}}, \quad y_{n+1}=\frac{1}{A_{2}+B_{2} x_{n}+y_{n}} \\
& \#(37,37): x_{n+1}=\frac{\alpha_{1}}{A_{1}+x_{n}+y_{n}}, \quad y_{n+1}=\frac{1}{A_{2}+B_{2} x_{n}+y_{n}} \\
& \#(12,39): x_{n+1}=\frac{\alpha_{1}}{x_{n}+y_{n}}, \quad y_{n+1}=\frac{x_{n}}{A_{2}+B_{2} x_{n}+y_{n}} \\
& \#(37,39): x_{n+1}=\frac{\alpha_{1}}{A_{1}+x_{n}+y_{n}}, \quad y_{n+1}=\frac{x_{n}}{A_{2}+B_{2} x_{n}+y_{n}} \\
& \#(12,44): x_{n+1}=\frac{\alpha_{1}}{x_{n}+y_{n}}, \quad y_{n+1}=\frac{\alpha_{2}+x_{n}}{A_{2}+B_{2} x_{n}+y_{n}} \\
& \#(37,44): x_{n+1}=\frac{\alpha_{1}}{A_{1}+x_{n}+y_{n}}, \quad y_{n+1}=\frac{\alpha_{2}+x_{n}}{A_{2}+B_{2} x_{n}+y_{n}}
\end{aligned}
$$

An important ingredient in understanding the global stability character of a system of difference equations, and thus for the special cases of System (1.1), is the boundedness nature of its solutions. Interestingly, all of the 18 special cases of System (1.1) have the boundedness characterization (B,B), that is, both components are always bounded. For the proofs of these see $[9],[14]$, and [15].

In addition to the boundedness characterization $(\mathrm{B}, \mathrm{B})$ of the solutions of System (1.1), we are interested in determining whether every solution converges to a finite limit, or every solution converges to a periodic solution.

Actually, for System (1.1) we offer the following conjecture.

Conjecture 1.1. Every solution of System (1.1) converges to a (not necessarily prime) period-two solution.

Conjecture 1.1 has been confirmed for System \#(12,12) in [37], and for System \# $(12,18)$ in $[15]$. In this paper we confirm Conjecture 1.1 for the 
following 9 special cases:

$$
\begin{gathered}
\#(12,11), \#(12,17), \#(12,32), \#(12,37), \#(37,11), \\
\#(37,12), \#(37,17), \#(37,32), \#(37,37) .
\end{gathered}
$$

Conjecture 1.1 has not yet been confirmed (or refuted) for the following 7 special cases:

$$
\begin{gathered}
\#(12,33), \#(12,39), \#(12,44), \#(37,18), \\
\#(37,33), \#(37,39), \#(37,44) .
\end{gathered}
$$

In our investigation the following theorems will play a crucial role.

Theorem 1.1. ([24] or [26]) Assume that $p$ and $q$ are real numbers. Then a necessary and sufficient condition for both roots of the equation

$$
\lambda^{2}+p \lambda+q=0
$$

to lie inside the unit circle is

$$
|p|<1+q<2 .
$$

Theorem 1.2. (Amleh, Camouzis, and Ladas, [1]) Let I be a set of real numbers and let

$$
F: I \times I \rightarrow I
$$

be a function $F(u, v)$ which increases in both variables. Then for every solution $\left\{x_{n}\right\}_{n=-1}^{\infty}$ of equation

$$
x_{n+1}=F\left(x_{n}, x_{n-1}\right), \quad n=0,1, \ldots
$$

the subsequences $\left\{x_{2 n}\right\}$ and $\left\{x_{2 n+1}\right\}$ of even and odd terms of the solution do exactly one of the following:

i) Eventually they are both monotonically increasing.

ii) Eventually they are both monotonically decreasing.

iii) One of them is monotonically increasing and the other is monotonically decreasing.

Theorem 1.3. (Camouzis and Ladas, [13]) Let I be a set of real numbers and let

$$
F: I \times I \rightarrow I
$$

be a function $F(u, v)$ which decreases in the first and increases in the second variable. Then for every solution $\left\{x_{n}\right\}_{n=-1}^{\infty}$ of equation

$$
x_{n+1}=F\left(x_{n}, x_{n-1}\right), \quad n=0,1, \ldots
$$

the subsequences $\left\{x_{2 n}\right\}$ and $\left\{x_{2 n+1}\right\}$ of even and odd terms of the solution do exactly one of the following:

i) They are both monotonically increasing.

ii) They are both monotonically decreasing. 
iii) Eventually, one of them is monotonically increasing and the other is monotonically decreasing.

The next theorem is a new global stability result which we employed to establish the global stability character of Systems \#(12,17), \#(37,17), \#(12,32), and \# $(37,32)$.

Theorem 1.4. Let

$$
x_{n+1}=f\left(x_{n}, x_{n-1}\right), \quad n=0,1, \ldots
$$

with

(1) $f \in C[(0, \infty) \times(0, \infty),(0, \infty)]$.

(2) $f(x, y)$ is decreasing in $x$ and $y$.

(3) $x f(x, x)$ is increasing in $x$.

(4) Equation (1.2) has a unique positive equilibrium point $\bar{x}$.

Then every positive solution $\left\{x_{n}\right\}_{n=-1}^{\infty}$ of Eq. (1.2) which is bounded from above and from below by positive constants converges to $\bar{x}$.

Proof. Let $\left\{x_{n}\right\}_{n=-1}^{\infty}$ be a positive solution of Eq. (1.2) which is bounded from above and from below by positive constants. Therefore we have that

$$
I=\liminf _{n \rightarrow \infty} x_{n} \text { and } S=\limsup _{n \rightarrow \infty} x_{n}
$$

both exist and are finite positive numbers.

We show that $I=S$. Assume, for the sake of contradiction, that $I<S$. For each $\varepsilon>0$ there exists $N=N(\varepsilon)$ such that for all $n \geq N$

$$
I-\varepsilon<x_{n}<S+\varepsilon \text {. }
$$

Therefore we have

$$
x_{n+1}<f(I-\varepsilon, I-\varepsilon), \text { for all } n \geq N
$$

and

$$
x_{n+1}>f(S+\varepsilon, S+\varepsilon), \text { for all } n \geq N .
$$

Since $\varepsilon$ is arbitrary the previous two inequalities become respectively

$$
S \leq f(I, I)
$$

and

$$
I \geq f(S, S) .
$$

From (1.3) and (1.4) we have

$$
S f(S, S) \leq S I \leq I f(I, I) \Rightarrow S f(S, S) \leq I f(I, I) \Leftrightarrow S \leq I
$$

which is a contradiction. 
Theorem 1.5. (Kulenovic, Ladas, and Sizer, [13] or [27]) Let $[a, b]$ be a closed and bounded interval of real numbers and let $F \in C\left([a, b]^{k+1},[a, b]\right)$ satisfy the following conditions:

(1) $F\left(z_{1}, \ldots, z_{k+1}\right)$ is monotonic in each of its arguments.

(2) For each $m, M \in[a, b]$ and for each $i \in\{1, \ldots, k+1\}$, we define

$$
M_{i}(m, M)=\left\{\begin{array}{lll}
M & \text { if } F \text { is increasing in } z_{i} \\
m & \text { if } F \text { is decreasing in } z_{i}
\end{array}\right.
$$

and

$$
m_{i}(m, M)=M_{i}(M, m)
$$

and we assume that if $(m, M)$ is a solution of the system:

$$
\left.\begin{array}{l}
M=F\left(M_{1}(m, M), \ldots, M_{k+1}(m, M)\right) \\
m=F\left(m_{1}(m, M), \ldots, m_{k+1}(m, M)\right)
\end{array}\right\},
$$

then $M=m$.

Then the difference equation

$$
y_{n+1}=F\left(y_{n}, y_{n-1}, \ldots, y_{n-k}\right), \quad n=0,1, \ldots
$$

has a unique equilibrium point $\bar{y} \in[a, b]$ and every solution of Eq. (1.5), with initial conditions in $[a, b]$, converges to $\bar{y}$.

For further reading on difference equations and systems of difference equations see [2], [4]-[8], [10], [11], [13], [16]-[26], [25], [28]-[36], [38].

$$
\text { 2. Systems \# }(12,11) \text { AND \# }(37,11)
$$

Consider the system

$$
x_{n+1}=\frac{\alpha_{1}}{A_{1}+x_{n}+y_{n}} \text { and } y_{n+1}=\frac{\alpha_{2}}{1+x_{n}}, \quad n=0,1, \ldots
$$

with

$$
\alpha_{1}, \alpha_{2}>0 \text { and } A_{1} \geq 0 \text {. }
$$

When

$$
A_{1}=0
$$

System (2.1) is System \#(12,11) and when

$$
A_{1}>0
$$

System $(2.1)$ is System \# $(37,11)$.

For System (2.1) we show that every solution has a finite limit.

System (2.1) has a unique equilibrium point $(\bar{x}, \bar{y})$, and $\bar{x}$ is the unique positive real root of the cubic equation

$$
\bar{x}^{3}+\left(A_{1}+1\right) \bar{x}^{2}+\left(A_{1}+\alpha_{2}-\alpha_{1}\right) \bar{x}-\alpha_{1}=0 .
$$


The characteristic equation of the linearized system of System (2.1) about $(\bar{x}, \bar{y})$ is

$$
\lambda^{2}+\frac{\alpha_{1}}{\left(A_{1}+\bar{x}+\bar{y}\right)^{2}} \lambda-\frac{\alpha_{1} \alpha_{2}}{(1+\bar{x})^{2}\left(A_{1}+\bar{x}+\bar{y}\right)^{2}}=0 .
$$

According to Theorem 1.1 a necessary and sufficient condition for both roots of the above equation to lie inside the unit circle is

$$
\frac{\alpha_{1}}{\left(A_{1}+\bar{x}+\bar{y}\right)^{2}}<1-\frac{\alpha_{1} \alpha_{2}}{(1+\bar{x})^{2}\left(A_{1}+\bar{x}+\bar{y}\right)^{2}}<2 .
$$

The right-hand side inequality of condition (2.2) is trivially true. For the left-hand side inequality of condition (2.2), using the equilibrium equations

$$
\bar{x}=\frac{\alpha_{1}}{A_{1}+\bar{x}+\bar{y}} \text { and } \bar{y}=\frac{\alpha_{2}}{1+\bar{x}},
$$

we have

$$
\begin{gathered}
\alpha_{1}(1+\bar{x})^{2}<(1+\bar{x})^{2}\left(A_{1}+\bar{x}+\bar{y}\right)^{2}-\alpha_{1} \alpha_{2} \\
\Leftrightarrow \bar{x}\left(A_{1}+\bar{x}+\bar{y}\right)(1+\bar{x})^{2}<(1+\bar{x})^{2}\left(A_{1}+\bar{x}+\bar{y}\right)^{2}-\bar{x}\left(A_{1}+\bar{x}+\bar{y}\right) \bar{y}(1+\bar{x}) \\
\Leftrightarrow \bar{x}(1+\bar{x})<(1+\bar{x})\left(A_{1}+\bar{x}+\bar{y}\right)-\bar{x} \bar{y} \\
\Leftrightarrow A_{1}(1+\bar{x})+\bar{y}>0
\end{gathered}
$$

which is true. It follows that $(\bar{x}, \bar{y})$ is locally asymptotically stable for all values of the parameters $\alpha_{1}, A_{1}$, and $\alpha_{2}$.

The boundedness character of System (2.1) is described by the following lemma. For its proof see [9] or [14].

Lemma 2.1. Both components $\left\{x_{n}\right\}$ and $\left\{y_{n}\right\}$ of System (2.1) are bounded from above and from below by positive constants.

By substituting the value of $y_{n}$ from the second equation of the system into the first we see that the component $\left\{x_{n}\right\}$ satisfies the second order rational difference equation

$$
\begin{aligned}
x_{n+1}=\frac{\alpha_{1}}{A_{1}+x_{n}+\frac{\alpha_{2}}{1+x_{n-1}}} & \\
& =\frac{\alpha_{1}+\alpha_{1} x_{n-1}}{A_{1}+\alpha_{2}+x_{n}+A_{1} x_{n-1}+x_{n} x_{n-1}}, n=1,2, \ldots .
\end{aligned}
$$

The function

$$
f(x, y)=\frac{\alpha_{1}+\alpha_{1} y}{A_{1}+\alpha_{2}+x+A_{1} y+x y}
$$

associated with Eq. (2.3) is decreasing in the first and increasing in the second argument. Then by employing Theorem 1.3 it follows that the subsequences $\left\{x_{2 n}\right\}$ and $\left\{x_{2 n+1}\right\}$ both converge to $\bar{x}$, since Eq. (2.3) has no prime period-two solutions. 
To establish that Eq. (2.3) has no prime period-two solutions we assume, for the sake of contradiction, that

$$
\ldots \phi, \psi, \phi, \psi, \ldots
$$

is a prime period-two solution of Eq. (2.3). Then

$$
\phi=\frac{\alpha_{1}+\alpha_{1} \phi}{A_{1}+\alpha_{2}+\psi+A_{1} \phi+\phi \psi} \text { and } \psi=\frac{\alpha_{1}+\alpha_{1} \psi}{A_{1}+\alpha_{2}+\phi+A_{1} \psi+\phi \psi}
$$

and so

$$
\alpha_{1}+\alpha_{1} \phi=\left(A_{1}+\alpha_{2}\right) \phi+\phi \psi+A_{1} \phi^{2}+\phi^{2} \psi
$$

and

$$
\alpha_{1}+\alpha_{1} \psi=\left(A_{1}+\alpha_{2}\right) \psi+\phi \psi+A_{1} \psi^{2}+\psi^{2} \phi
$$

By subtracting (2.5) from (2.4) and then by dividing the result by $\phi-\psi$ we obtain

$$
\alpha_{1}=A_{1}+\alpha_{2}+A_{1}(\phi+\psi)+\phi \psi .
$$

By substituting the value of $\alpha_{1}$ from (2.6) into (2.4) we obtain

$$
A_{1}+\alpha_{2}+A_{1} \phi+A_{1} \psi+A_{1} \phi \psi=0
$$

which is a contradiction.

According to the preceding discussion the next result follows.

Theorem 2.1. The unique equilibrium point of System (2.1) is globally asymptotically stable.

For an alternative proof of Theorem 2.1 see Section 6 .

$$
\text { 3. Systems \# }(12,17) \text { AND \# }(37,17)
$$

Consider the system

$$
x_{n+1}=\frac{\alpha_{1}}{A_{1}+x_{n}+y_{n}} \text { and } y_{n+1}=\frac{x_{n}}{A_{2}+x_{n}}, \quad n=0,1, \ldots
$$

with

$$
\alpha_{1}, A_{2}>0 \text { and } A_{1} \geq 0 \text {. }
$$

When

$$
A_{1}=0
$$

System $(3.1)$ is System \# $(12,17)$ and when

$$
A_{1}>0
$$

System $(3.1)$ is System \# $(37,17)$.

For System (3.1) we show that every solution has a finite limit.

System (3.1) has a unique equilibrium point $(\bar{x}, \bar{y})$, and $\bar{x}$ is the unique positive real root of the cubic equation

$$
\bar{x}^{3}+\left(A_{1}+A_{2}+1\right) \bar{x}^{2}+\left(A_{1} A_{2}-\alpha_{1}\right) \bar{x}-\alpha_{1} A_{2}=0 .
$$


The characteristic equation of the linearized system of System (3.1) about $(\bar{x}, \bar{y})$ is

$$
\lambda^{2}+\frac{\alpha_{1}}{\left(A_{1}+\bar{x}+\bar{y}\right)^{2}} \lambda+\frac{\alpha_{1} A_{2}}{\left(A_{2}+\bar{x}\right)^{2}\left(A_{1}+\bar{x}+\bar{y}\right)^{2}}=0 .
$$

According to Theorem 1.1 a necessary and sufficient condition for both roots of the above equation to lie inside the unit circle is

$$
\frac{\alpha_{1}}{\left(A_{1}+\bar{x}+\bar{y}\right)^{2}}<1+\frac{\alpha_{1} A_{2}}{\left(A_{2}+\bar{x}\right)^{2}\left(A_{1}+\bar{x}+\bar{y}\right)^{2}}<2 .
$$

By using the equilibrium equations

$$
\bar{x}=\frac{\alpha_{1}}{A_{1}+\bar{x}+\bar{y}}
$$

and

$$
\bar{y}=\frac{\bar{x}}{A_{2}+\bar{x}}
$$

condition (3.2) can be written as

$$
\frac{\bar{x}^{2}}{\alpha_{1}}<1+\frac{A_{2}}{\alpha_{1}} \bar{y}^{2}<2 .
$$

From (3.3) we have

$$
\begin{gathered}
\bar{x}^{2}=\frac{\alpha_{1}^{2}}{\left(A_{1}+\bar{x}+\bar{y}\right)^{2}}<\frac{\alpha_{1}^{2}}{\bar{x}^{2}} \Rightarrow \bar{x}^{4}<\alpha_{1}^{2} \\
\Rightarrow \bar{x}^{2}<\alpha_{1}<\alpha_{1}+A_{2} \bar{y}^{2} \Rightarrow \frac{\bar{x}^{2}}{\alpha_{1}}<1+\frac{A_{2}}{\alpha_{1}} \bar{y}^{2},
\end{gathered}
$$

and so the left-hand side inequality of (3.5) holds. Using now both (3.3) and (3.4) we have

$$
\begin{gathered}
\bar{x}^{2}=\frac{\alpha_{1}^{2}}{\left(A_{1}+\bar{x}+\bar{y}\right)^{2}} \Rightarrow \bar{y}^{2}\left(A_{2}+\bar{x}\right)^{2}=\frac{\alpha_{1}^{2}}{\left(A_{1}+\bar{x}+\bar{y}\right)^{2}} \\
\Rightarrow \bar{y}^{2}=\frac{\alpha_{1}^{2}}{\left(A_{2}+\bar{x}\right)^{2}\left(A_{1}+\bar{x}+\bar{y}\right)^{2}}<\frac{\alpha_{1}^{2}}{A_{2}^{2} \bar{y}^{2}} \\
\Rightarrow A_{2}^{2} \bar{y}^{4}<\alpha_{1}^{2} \Rightarrow \frac{A_{2}}{\alpha_{1}} \bar{y}^{2}<1,
\end{gathered}
$$

and so the right-hand side inequality of (3.5) holds. It follows that $(\bar{x}, \bar{y})$ is locally asymptotically stable for all values of the parameters $\alpha_{1}, A_{1}$, and $A_{2}$.

The boundedness character of System (3.1) is described by the following lemma. For its proof see [9] or [14].

Lemma 3.1. Both components $\left\{x_{n}\right\}$ and $\left\{y_{n}\right\}$ of System (3.1) are bounded from above and from below by positive constants. 
By substituting the value of $y_{n}$ from the second equation of the system into the first we see that the component $\left\{x_{n}\right\}$ satisfies the second order rational difference equation

$$
\begin{aligned}
x_{n+1}= & \frac{\alpha_{1}}{A_{1}+x_{n}+\frac{x_{n-1}}{A_{2}+x_{n-1}}} \\
& =\frac{\alpha_{1} A_{2}+\alpha_{1} x_{n-1}}{A_{1} A_{2}+A_{2} x_{n}+\left(1+A_{1}\right) x_{n-1}+x_{n} x_{n-1}}, n=1,2, \ldots .
\end{aligned}
$$

The function

$$
f(x, y)=\frac{\alpha_{1} A_{2}+\alpha_{1} y}{A_{1} A_{2}+A_{2} x+\left(1+A_{1}\right) y+x y}
$$

associated with Eq. (3.6) is decreasing in both variables and the function $x f(x, x)$ is increasing. Therefore, in view of Lemma 3.1 and by Theorem 1.4 it follows, that every positive solution of Eq. (3.6) converges to $\bar{x}$. From this and the second equation of the system the next theorem follows.

Theorem 3.1. The unique equilibrium point of System (3.1) is globally asymptotically stable.

$$
\text { 4. Systems \#(12,32) And \#(37,32) }
$$

Consider the system

$$
x_{n+1}=\frac{\alpha_{1}}{A_{1}+x_{n}+y_{n}} \text { and } y_{n+1}=\frac{\alpha_{2}+\beta_{2} x_{n}}{1+x_{n}}, n=0,1, \ldots
$$

with

$$
\alpha_{1}, \alpha_{2}, \beta_{2}>0 \text { and } A_{1} \geq 0
$$

When

$$
A_{1}=0
$$

System (4.1) is System \# $(12,32)$ and when

$$
A_{1}>0
$$

System (4.1) is System \# $(37,32)$.

For System (4.1) we show that every solution has a finite limit.

When $\alpha_{2}=\beta_{2}$, System (4.1) reduces to

$$
x_{n+1}=\frac{\alpha_{1}}{A_{1}+\alpha_{2}+x_{n}} \text { and } y_{n+1}=\alpha_{2}, \quad n=1,2, \ldots,
$$

for which every solution converges to a finite number. In the sequel, we consider the case where $\alpha_{2} \neq \beta_{2}$.

System (4.1) has a unique equilibrium point $(\bar{x}, \bar{y})$, and $\bar{x}$ is the unique positive real root of the cubic equation

$$
\bar{x}^{3}+\left(A_{1}+\beta_{2}+1\right) \bar{x}^{2}+\left(A_{1}+\alpha_{2}-\alpha_{1}\right) \bar{x}-\alpha_{1}=0 .
$$


The characteristic equation of the linearized system of System (4.1) about $(\bar{x}, \bar{y})$ is

$$
\lambda^{2}+\frac{\alpha_{1}}{\left(A_{1}+\bar{x}+\bar{y}\right)^{2}} \lambda+\frac{\left(\beta_{2}-\alpha_{2}\right) \alpha_{1}}{(1+\bar{x})^{2}\left(A_{1}+\bar{x}+\bar{y}\right)^{2}}=0 .
$$

According to Theorem 1.1 a necessary and sufficient condition for both roots of the above equation to lie inside the unit circle is

$$
\frac{\alpha_{1}}{\left(A_{1}+\bar{x}+\bar{y}\right)^{2}}<1+\frac{\alpha_{1}\left(\beta_{2}-\alpha_{2}\right)}{(1+\bar{x})^{2}\left(A_{1}+\bar{x}+\bar{y}\right)^{2}}<2 .
$$

By using the equilibrium equations

$$
\bar{x}=\frac{\alpha_{1}}{A_{1}+\bar{x}+\bar{y}}
$$

and

$$
\bar{y}=\frac{\alpha_{2}+\beta_{2} \bar{x}}{1+\bar{x}}
$$

condition (4.3) can be written as

$$
\frac{\bar{x}^{2}}{\alpha_{1}}<1+\frac{\left(\beta_{2}-\alpha_{2}\right) \bar{x}^{2}}{\alpha_{1}(1+\bar{x})^{2}}<2
$$

For the left-hand side inequality of (4.6) we have

$$
\begin{gathered}
\bar{x}^{2}(1+\bar{x})^{2}<\alpha_{1}(1+\bar{x})^{2}+\left(\beta_{2}-\alpha_{2}\right) \bar{x}^{2} \\
\Leftrightarrow \bar{x}^{2}(1+\bar{x})^{2}<\alpha_{1}+\alpha_{1} \bar{x}^{2}+2 \alpha_{1} \bar{x}+\left(\beta_{2}-\alpha_{2}\right) \bar{x}^{2}
\end{gathered}
$$

and then by using the value of $\alpha_{1}$ from (4.2) we have

$$
\begin{aligned}
\bar{x}^{2}(1+\bar{x})^{2} & <\bar{x}^{3}+\left(A_{1}+\beta_{2}+1\right) \bar{x}^{2}+\left(A_{1}+\alpha_{2}-\alpha_{1}\right) \bar{x}+\alpha_{1} \bar{x}^{2}+2 \alpha_{1} \bar{x}+\beta_{2} \bar{x}^{2}-\alpha_{2} \bar{x}^{2} \\
& \Leftrightarrow \bar{x}^{3}+\bar{x}^{2}<A_{1} \bar{x}+2 \beta_{2} \bar{x}+A_{1}+\alpha_{2}+\alpha_{1} \bar{x}+\alpha_{1}-\alpha_{2} \bar{x} .
\end{aligned}
$$

Then by using again the value of $\alpha_{1}$ from (4.2) the above inequality can be written as

$$
\begin{gathered}
\bar{x}^{3}+\bar{x}^{2}<A_{1} \bar{x}+2 \beta_{2} \bar{x}+A_{1}+\alpha_{2}+\alpha_{1} \bar{x}+\bar{x}^{3}+\left(A_{1}+\beta_{2}+1\right) \bar{x}^{2}+\left(A_{1}+\alpha_{2}-\alpha_{1}\right) \bar{x}-\alpha_{2} \bar{x} \\
\Leftrightarrow 2\left(A_{1}+\beta_{2}\right) \bar{x}+A_{1}+\alpha_{2}+\left(A_{1}+\beta_{2}\right) \bar{x}^{2}>0
\end{gathered}
$$

which is true. For the right-hand side inequality of (4.6) we have

$$
\left(\beta_{2}-\alpha_{2}\right) \bar{x}^{2}<\alpha_{1}(1+\bar{x})^{2} \Leftrightarrow\left(\beta_{2}-\alpha_{2}\right) \bar{x}^{2}<\alpha_{1}+2 \alpha_{1} \bar{x}+\alpha_{1} \bar{x}^{2}
$$

and then by using the value of $\alpha_{1}$ from (4.2) we have

$$
\begin{gathered}
\left(\beta_{2}-\alpha_{2}\right) \bar{x}^{2}<\bar{x}^{3}+\left(A_{1}+\beta_{2}+1\right) \bar{x}^{2}+\left(A_{1}+\alpha_{2}-\alpha_{1}\right) \bar{x}+2 \alpha_{1} \bar{x}+\alpha_{1} \bar{x}^{2} \\
\Leftrightarrow-\alpha_{2} \bar{x}^{2}<\bar{x}^{3}+\left(A_{1}+\alpha_{1}+1\right) \bar{x}^{2}+\left(A_{1}+\alpha_{1}+\alpha_{2}\right) \bar{x}
\end{gathered}
$$

which is true. It follows that $(\bar{x}, \bar{y})$ is locally asymptotically stable for all values of the parameters $\alpha_{1}, A_{1}, \alpha_{2}$, and $\beta_{2}$. 
The boundedness character of System (4.1) is described by the following lemma. For its proof see [9].

Lemma 4.1. Both components $\left\{x_{n}\right\}$ and $\left\{y_{n}\right\}$ of System (4.1) are bounded from above and from below by positive constants.

By substituting the value of $y_{n}$ from the second equation of the system into the first we see that the component $\left\{x_{n}\right\}$ satisfies the second order rational difference equation

$$
\begin{aligned}
x_{n+1}= & \frac{\alpha_{1}}{A_{1}+x_{n}+\frac{\alpha_{2}+\beta_{2} x_{n-1}}{1+x_{n-1}}} \\
& =\frac{\alpha_{1}+\alpha_{1} x_{n-1}}{A_{1}+\alpha_{2}+x_{n}+\left(A_{1}+\beta_{2}\right) x_{n-1}+x_{n} x_{n-1}}, n=1,2, \ldots .
\end{aligned}
$$

The function

$$
f(x, y)=\frac{\alpha_{1}+\alpha_{1} y}{A_{1}+\alpha_{2}+x+\left(A_{1}+\beta_{2}\right) y+x y}
$$

associated with Eq. (4.7) is decreasing in both variables when

$$
\alpha_{2}<\beta_{2}
$$

and is decreasing in the first and increasing in the second argument when

$$
\alpha_{2}>\beta_{2} \text {. }
$$

When (4.8) holds, and because the function $x f(x, x)$ is increasing, by employing Theorem 1.4 it follows, that every solution of Eq. (4.7) converges to $\bar{x}$. Alternatively, when (4.8) holds we can arrive at the same result by employing Theorem 1.5 to the Eq. (4.7) in the interval $\left[0, \frac{\alpha_{1}}{A_{1}+\alpha_{2}}\right]$.

When (4.9) holds, by employing Theorem 1.3 it follows that the subsequences $\left\{x_{2 n}\right\}$ and $\left\{x_{2 n+1}\right\}$ both converge to $\bar{x}$, since Eq. (4.7) has no prime period-two solutions. The proof that Eq. (4.7) has no prime period-two solutions is similar to the proof of the fact that Eq. (2.3) has no prime period-two solutions and will be omitted.

From the preceding discussion the next result follows.

Theorem 4.1. The unique equilibrium point of System (4.1) is globally asymptotically stable.

\section{System \# $(12,37)$}

Consider the system

$$
x_{n+1}=\frac{\alpha}{x_{n}+y_{n}} \text { and } y_{n+1}=\frac{1}{A+B x_{n}+y_{n}}, n=0,1, \ldots .
$$

For this system we show that every solution has a finite limit. 
The equilibrium points of System (5.1) are the points $(\bar{x}, \bar{y}) \in(0, \infty)^{2}$ such that:

and

$$
\bar{x}=\frac{\alpha}{\bar{x}+\bar{y}}
$$

From (5.2) we have

$$
\bar{y}=\frac{1}{A+B \bar{x}+\bar{y}} .
$$

from which it follows that

$$
\begin{gathered}
\bar{x}=\frac{\alpha}{\bar{x}+\bar{y}} \Rightarrow \bar{x}^{2}+\bar{x} \bar{y}=\alpha \Rightarrow \\
\bar{y}=\frac{\alpha-\bar{x}^{2}}{\bar{x}}>0,
\end{gathered}
$$

$$
\bar{x}<\sqrt{\alpha} .
$$

Furthermore, from (5.3) we have

$$
\bar{y}=\frac{1}{A+B \bar{x}+\bar{y}}<\frac{1}{\bar{y}}
$$

from which it follows that

$$
\bar{y}<1 .
$$

By substituting the value of $\bar{y}$ from (5.4) into (5.3) we see that the component $\bar{x}$ satisfies the following quartic polynomial:

$$
h(\bar{x})=(1-B) \bar{x}^{4}-A \bar{x}^{3}+(B \alpha-2 \alpha-1) \bar{x}^{2}+A \alpha \bar{x}+\alpha^{2}=0 .
$$

When $B \geq 1$ according to the Descartes Rule of Signs the above quartic polynomial has a unique positive real root. When $B<1$ the coefficients of the above polynomial change sign twice and therefore has two or none positive real roots. But $h(0)=\alpha^{2}>0$ and $h(\sqrt{\alpha})=-\alpha<0$ which means that the equation $h(\bar{x})=0$ has a unique positive real root less than $\sqrt{\alpha}$. Hence, System (5.1) has a unique equilibrium point $(\bar{x}, \bar{y})$.

The characteristic equation of the linearized system of System (5.1) about $(\bar{x}, \bar{y})$ is

$$
\lambda^{2}+\left(\frac{\alpha}{(\bar{x}+\bar{y})^{2}}+\frac{1}{(A+B \bar{x}+\bar{y})^{2}}\right) \lambda+\frac{\alpha-\alpha B}{(\bar{x}+\bar{y})^{2}(A+B \bar{x}+\bar{y})^{2}}=0 .
$$

According to Theorem 1.1 a necessary and sufficient condition for both roots of the above equation to lie inside the unit circle is

$$
\frac{\alpha}{(\bar{x}+\bar{y})^{2}}+\frac{1}{(A+B \bar{x}+\bar{y})^{2}}<1+\frac{\alpha-\alpha B}{(\bar{x}+\bar{y})^{2}(A+B \bar{x}+\bar{y})^{2}}<2 .
$$

By using (5.2), (5.3), and (5.4) condition (5.8) can be written as

$$
\frac{\bar{x}^{2}}{\alpha}+\frac{\left(\alpha-\bar{x}^{2}\right)^{2}}{\bar{x}^{2}}<1+\frac{(1-B)}{\alpha} \bar{x}^{2} \bar{y}^{2}<2 .
$$


For the left-hand side inequality of (5.9), using again (5.4), we have

$$
\frac{\bar{x}^{2}}{\alpha}+\frac{\left(\alpha-\bar{x}^{2}\right)^{2}}{\bar{x}^{2}}<1+\frac{(1-B)}{\alpha}\left(\alpha-\bar{x}^{2}\right)^{2} \Leftrightarrow
$$

$\bar{x}^{4}+\alpha^{3}+\alpha \bar{x}^{4}-2 \alpha^{2} \bar{x}^{2}<\alpha \bar{x}^{2}+\alpha^{2} \bar{x}^{2}+(1-B) \bar{x}^{4} \bar{x}^{2}-2 \alpha \bar{x}^{4}-B \alpha^{2} \bar{x}^{2}+2 \alpha B \bar{x}^{4}$

which by substituting the value of $(1-B) \bar{x}^{4}$ from (5.7) into it can be written as

$$
\alpha^{3}+\alpha\left[(B \alpha-2 \alpha-1) \bar{x}^{2}+(1-B) \bar{x}^{4}\right]<A \bar{x}^{5}-A \alpha \bar{x}^{3} .
$$

Then by substituting the value of $\left[(B \alpha-2 \alpha-1) \bar{x}^{2}+(1-B) \bar{x}^{4}\right]$ from (5.7) into the above inequality we obtain

$$
\alpha \bar{x}^{3}-\alpha^{2} \bar{x}<\bar{x}^{5}-\alpha \bar{x}^{3} \Leftrightarrow \alpha \bar{x}^{2}-\alpha^{2}<\bar{x}^{4}-\alpha \bar{x}^{2} \Leftrightarrow\left(\bar{x}^{2}-\alpha\right)^{2}>0
$$

which is true. For the right-hand side inequality of (5.9) we have

$$
(1-B) \bar{x}^{2} \bar{y}^{2}<\alpha
$$

which clearly holds when $B \geq 1$. Next we assume that $B<1$. From (5.5) and (5.6) it follows that

$$
\bar{x}^{2} \bar{y}^{2}<\alpha \Rightarrow(1-B) \bar{x}^{2} \bar{y}^{2}<\alpha(1-B)<\alpha .
$$

It follows that $(\bar{x}, \bar{y})$ is locally asymptotically stable for all values of the parameters $\alpha, A$, and $B$.

The boundedness character of System (5.1) is described by the following lemma. For its proof see [14].

Lemma 5.1. Both components $\left\{x_{n}\right\}$ and $\left\{y_{n}\right\}$ of System \#(12,37) are bounded from above and from below by positive constants.

In view of Lemma 5.1 we have that

$$
I_{x}=\liminf _{n \rightarrow \infty} x_{n}, \quad S_{x}=\limsup _{n \rightarrow \infty} x_{n}
$$

and

$$
I_{y}=\liminf _{n \rightarrow \infty} y_{n}, \quad S_{y}=\limsup _{n \rightarrow \infty} y_{n}
$$

all exist and are finite positive numbers. For the proof of the following result, see Section 6 .

Theorem 5.1. The unique equilibrium point of System (5.1) is globally asymptotically stable. 
Consider the system

$x_{n+1}=\frac{\alpha_{1}}{A_{1}+B_{1} x_{n}+C_{1} y_{n}}$ and $y_{n+1}=\frac{\alpha_{2}}{A_{2}+B_{2} x_{n}+C_{2} y_{n}}, n=0,1, \ldots$.

System (6.1) was first studied by Camouzis and Ladas in [14] where they showed that every solution converges to a not necessarily prime period two solution. We actually show here that every solution converges to a finite limit.

Clearly, both components are bounded from above and from below by positive constants. Therefore,

$$
I_{x}=\liminf _{n \rightarrow \infty} x_{n}, \quad S_{x}=\limsup _{n \rightarrow \infty} x_{n}
$$

and

$$
I_{y}=\liminf _{n \rightarrow \infty} y_{n}, \quad S_{y}=\limsup _{n \rightarrow \infty} y_{n}
$$

all exist and are finite positive numbers.

Here, the functions

$$
f(x, y)=\frac{\alpha_{1}}{A_{1}+B_{1} x+C_{1} y} \text { and } g(x, y)=\frac{\alpha_{2}}{A_{2}+B_{2} x+C_{2} y}
$$

associated with System (6.1) decrease in their variables.

Theorem 6.1. Every solution of System (6.1) converges to a finite limit.

Proof. For each $\varepsilon>0$ there exist $N_{1}=N_{1}(\varepsilon)$ and $N_{2}=N_{2}(\varepsilon)$ such that

$$
I_{x}-\varepsilon<x_{n}<S_{x}+\varepsilon, \text { for all } n \geq N_{1}
$$

and

$$
I_{y}-\varepsilon<y_{n}<S_{y}+\varepsilon, \text { for all } n \geq N_{2} .
$$

In view of the monotonic character of $f$ we have

$$
x_{n+1}=f\left(x_{n}, y_{n}\right)<f\left(I_{x}-\varepsilon, I_{y}-\varepsilon\right), \text { for all } n \geq N
$$

where $N=\max \left\{N_{1}, N_{2}\right\}$.

Since $\varepsilon$ is arbitrary we have

$$
S_{x} \leq f\left(I_{x}, I_{y}\right)
$$

or

$$
S_{x} \leq \frac{\alpha_{1}}{A_{1}+B_{1} I_{x}+C_{1} I_{y}}
$$

Similarly,

$$
I_{x} \geq \frac{\alpha_{1}}{A_{1}+B_{1} S_{x}+C_{1} S_{y}},
$$


and

$$
S_{y} \leq \frac{\alpha_{1}}{A_{2}+B_{2} I_{x}+C_{2} I_{y}},
$$

$$
I_{y} \geq \frac{\alpha_{2}}{A_{2}+B_{2} S_{x}+C_{2} S_{y}} .
$$

From (6.4) and (6.5) we have

$$
0 \leq A_{1}\left(S_{x}-I_{x}\right) \leq C_{1}\left(I_{x} S_{y}-S_{x} I_{y}\right) .
$$

From (6.6) and (6.7) we have

$$
0 \leq A_{2}\left(S_{y}-I_{y}\right) \leq B_{2}\left(S_{x} I_{y}-I_{x} S_{y}\right) .
$$

In view of (6.8) and (6.9) the result follows.

Assuming that we relax the condition on the parameters so that,

$$
A_{1} \geq 0 \text { and } C_{2}=0
$$

the proof of Theorem 6.1 still holds implying that every solution of System (2.1) converges to a finite limit.

Also, when

$$
A_{1}=0
$$

the proof of Theorem 6.1 still holds implying that every solution of System (5.1) converges to a finite limit.

\section{REFERENCES}

[1] A. M. Amleh, E. Camouzis, and G. Ladas, On the dynamics of a rational difference equation, Part 1, Int. J. Difference Equ., 3 (2008), 1-35.

[2] A. M. Amleh, E. Camouzis, and G. Ladas, On the dynamics of a rational difference equation, Part 2, Int. J. Difference Equ., 3 (2008), 195-225.

[3] A. M. Amleh, E. Camouzis, G. Ladas, and M. Radin, Patterns of boundedness of a rational system in the plane, J. Difference Equ. Appl., 16 (2010), 1197-1236.

[4] A. Brett, E. Camouzis, C. Lynd, and G. Ladas, On the boundedness character of a rational system, JNMaS, 1 (2009), 1-10.

[5] E. Camouzis, Boundedness of solutions of a rational system of difference equations, Proceedings of the 14th International Conference on Difference Equations and Applications held in Instanbul, Turkey, July 21-25, 2008, Ugur-Bahcesehir University Publishing Company, Istanbul, Turkey, Difference Equations and Applications, ISBN 978-975-6437-80-3 (2009), 157-164 .

[6] E. Camouzis, E. Drymonis, and G. Ladas, On the global character of the system $x_{n+1}=\frac{\alpha}{x_{n}+y_{n}}$ and $y_{n+1}=\frac{y_{n}}{B x_{n}+y_{n}}$, Commun. Appl. Nonlinear Anal., 16 (2009), $51-64$.

[7] E. Camouzis, E. Drymonis, and G. Ladas, Patterns of boundedness of the rational system $x_{n+1}=\frac{\alpha_{1}+\beta_{1} x_{n}}{A_{1}+C_{1} y_{n}}$ and $y_{n+1}=\frac{\alpha_{2}+\beta_{2} x_{n}+\gamma_{2} y_{n}}{A_{2}+B_{2} x_{n}+y_{n}}$, Fasc. Math., 44 (2010), 9-18.

[8] E. Camouzis, E. Drymonis, and G. Ladas, Patterns of boundedness of the rational system $x_{n+1}=\frac{\alpha_{1}+\beta_{1} x_{n}}{A_{1}+B_{1} x_{n}+C_{1} y_{n}}$ and $y_{n+1}=\frac{\alpha_{2}+\beta_{2} x_{n}+\gamma_{2} y_{n}}{A_{2}+B_{2} x_{n}+C_{2} y_{n}}$, Commun. Appl. Nonlinear Anal., 18 (2011), 1-23. 
[9] E. Camouzis, E. Drymonis, G. Ladas, and W. Tikjha, Patterns of boundedness of the rational system $x_{n+1}=\frac{\alpha_{1}}{A_{1}+B_{1} x_{n}+C_{1} y_{n}}$ and $y_{n+1}=\frac{\alpha_{2}+\beta_{2} x_{n}+\gamma_{2} y_{n}}{A_{2}+B_{2} x_{n}+y_{n}}$, J. Difference Equ. Appl., 18 (2012), 89-110.

[10] E. Camouzis, A. Gilbert, M. Heissan, and G. Ladas, On the boundedness character of the system $x_{n+1}=\frac{\alpha_{1}+\gamma_{1} y_{n}}{x_{n}}$ and $y_{n+1}=\frac{\alpha_{2}+\beta_{2} x_{n}+\gamma_{2} y_{n}}{A_{2}+x_{n}+y_{n}}$, Commun. Math. Anal., 7 (2009), 41-50.

[11] E. Camouzis, C. M. Kent, G. Ladas, and C. D. Lynd, On the global character of solutions of the system $x_{n+1}=\frac{\alpha_{1}+y_{n}}{x_{n}}$ and $y_{n+1}=\frac{\alpha_{2}+\beta_{2} x_{n}+\gamma_{2} y_{n}}{A_{2}+B_{2} x_{n}+C_{2} y_{n}}$, J. Difference Equ. Appl., (2011).

[12] E. Camouzis, M. R. S. Kulenović, G. Ladas, and O. Merino, Rational systems in the plane, J. Difference Equ. Appl., 15 (2009), 303-323.

[13] E. Camouzis and G. Ladas, Dynamics of Third-Order Rational Difference Equations; With Open Problems and Conjectures, Chapman \& Hall/CRC Press, November 2007.

[14] E. Camouzis and G. Ladas, Global results on rational systems in the plane, I, J. Difference. Equ. Appl., 16 (2010), 975-1013.

[15] E. Camouzis, G. Ladas, and L. Wu, On the global character of the system $x_{n+1}=$ $\frac{\alpha_{1}+\gamma_{1} y_{n}}{x_{n}}$ and $y_{n+1}=\frac{\beta_{2} x_{n}+\gamma_{2} y_{n}}{B_{2} x_{n}+C_{2} y_{n}}$, Int. J. Pure Appl. Math., 53 (2009), 21-36.

[16] D. Clark and M. R. S. Kulenović, On a coupled system of rational difference equations, Comput. Math. Appl., 43 (2002), 849-867.

[17] D. Clark, M. R. S. Kulenović, and J. F. Selgrade, Global asymptotic behavior of a two dimensional difference equation modelling competition, Nonlinear Anal. TMA, 52 (2003), 1765-1776.

[18] C. A. Clark, M. R. S. Kulenović, and J. F. Selgrade, On a system of rational difference equations, J. Difference Equ. Appl., 11 (2005), 565-580.

[19] J. M. Cushing, S. Levarge, N. Chitnis and S. M. Henson, Some discrete competition models and the competitive exclusion principle, J. Difference Equ. Appl., 10 (2004), 1139-1152.

[20] E. Drymonis, Y. Kostrov, and Z. Kudlak, On rational difference equations with nonnegative periodic coefficients, Int. J. Difference Equ., 7 (2012).

[21] S. Elaydi, and R. J. Sacker, Global Stability of Periodic Orbits of Nonautonomous Difference Equations in Population Biology and the Cushing-Henson Conjectures, Proceedings of the Eighth International Conference on Difference Equations and Applications, Chapman \& Hall/CRC (2003), pp. 112-126.

[22] M. Garić-Demirović, M. R. S. Kulenović and M. Nurkanović, Global behavior of four competitive rational systems of difference equations on the plane, Discrete Dyn. Nat. Soc., 2009, Art. ID 153058, 34 pp.

[23] E. A. Grove, Y. Kostrov, M. A. Radin, and S. Schultz, On the global character of solutions of the system $x_{n+1}=\frac{\alpha_{1}}{x_{n}+y_{n}}$ and $y_{n+1}=\frac{\alpha_{2}+\beta_{2} x_{n}}{B_{2} x_{n}+y_{n}}$, Commun. Appl. Nonlinear Anal., 17 (2010), 69-81.

[24] E.A. Grove and G. Ladas, Periodicities in Nonlinear Difference Equations, Chapman \& Hall/CRC Press, 2005.

[25] S. Kalabusić, M. R. S. Kulenović and E. Pilav, Global dynamics of a system of rational difference equations in the plane, Adv. Difference Equ., 2009, Art. ID 132802, $30 \mathrm{pp}$.

[26] V. L. Kocic and G. Ladas, Global Behavior of Nonlinear Difference Equations of Higher Order with Applications, Kluwer Academic Publishers, Dordrecht, 1993. 
[27] M. R. S. Kulenović, G. Ladas, and W. S. Sizer, On the recursive sequence $x_{n+1}=$ $\frac{\alpha x_{n}+\beta x_{n-1}}{\gamma x_{n}+C x_{n-1}}$, Math. Sci. Res. Hot-Line, 2 (5) (1998), 1-16.

[28] M. R. S. Kulenović and O. Merino, Competitive-exclusion versus competitivecoexistence for systems in the plane, Discrete Contin. Dyn. Syst. Ser. B, 6 (2006), $1141-1156$.

[29] M.R.S. Kulenović and G. Ladas, Dynamics of Second Order Rational Difference Equations; with Open Problems and Conjectures, Chapman \& Hall/CRC Press, 2001.

[30] M. R. S. Kulenović and M. Nurkanović, Asymptotic behavior of two dimensional linear fractional system of difference equations, Rad. Mat., 11 (2002), 59-78.

[31] M. R. S. Kulenović and M. Nurkanović, Asymptotic behavior of a linear fractional system of difference equations, J. Inequal. Appl., (2005), 127-144.

[32] M. R. S. Kulenović and M. Nurkanović, Asymptotic behavior of a competitive system of linear fractional difference equations, Adv. Difference Equ., 3 (2006), 1-13.

[33] G. Ladas, Open problems and conjectures, J. Difference Equ. Appl., 1 (4) (1995), 413-419.

[34] G. Ladas, G. Tzanetopoulos, and A. Tovbis, On May's host parasitoid model, J. Difference Equ. Appl., 2 (1996), 195-204.

[35] G. Lugo and F. J. Palladino, On the boundedness character of rational systems in the plane, J. Difference Equ. Appl., (2011), 1-11

[36] G. Lugo and F. J. Palladino, Private Communication.

[37] E. Magnucka-Blandzi and J. Popenda, On the asymptotic behavior of a rational system of difference equations, J. Difference Equ. Appl., 5 (3) (1999), 271-286.

[38] R. M. May, Host-Parasitoid system in patchy environments, A phenomenological model, Journal of Animal Ecology, 47 (1978), 833-843.

(Received: May 6, 2012)

(Revised: July 6, 2012)
University of Rhode Island

Department of Mathematics

Kingston, RI 02881-0816, USA

E-mail: mdrymonis@math.uri.edu gladas@math.uri.edu 